Review Article

\title{
Ophthalmic Metastasis of Breast Cancer and Ocular Side Effects from Breast Cancer Treatment and Management: Mini Review
}

\author{
Ilias Georgalas, Theodore Paraskevopoulos, Chryssanthi Koutsandrea, Evgenia Kardara, \\ Panagiotis Malamos, Dimitrios Ladas, and Dimitris Papaconstantinou
}

Department of Ophthalmology, “G. Gennimatas” Hospital of Athens, University of Athens, 154 Mesogeion Avenue, 11527 Athens, Greece

Correspondence should be addressed to Ilias Georgalas; igeorgalas@yahoo.com

Received 8 November 2014; Revised 1 April 2015; Accepted 12 April 2015

Academic Editor: Stephen H. Safe

Copyright (c) 2015 Ilias Georgalas et al. This is an open access article distributed under the Creative Commons Attribution License, which permits unrestricted use, distribution, and reproduction in any medium, provided the original work is properly cited.

Breast cancer is one of the most common malignant diseases occurring in women, and its incidence increases over the years. It is the main site of origin in ocular metastatic disease in women, and, due to its hematogenous nature of metastatic spread, it affects mainly the uveal tissue. The purpose of this paper is to summarize the clinical manifestations of the breast cancer ocular metastatic disease, alongside the side effects of the available treatment options for the management and regression of the systematic and ophthalmic disease.

\section{Introduction and Epidemiology}

Breast cancer is the most common neoplastic disorder diagnosed in women [1]. It is classified as the second most frequent cause of death in women, after lung cancer [1]. Despite recent advances in early diagnosis and effective treatment, it is estimated that up to one-third of patients having been diagnosed with breast cancer will develop metastatic disease [2]. In contrast, breast cancer in men, male breast cancer (MBC), is rare and accounts for less than $1 \%$ of total neoplastic cases in male population and about $1 \%$ of all breast cancer diagnoses [3, 4]. Male breast cancer's incidence is increasing over the years, due to lack of awareness of male population regarding this disease. As a result, male patients are diagnosed in a more advanced stage of the disease [5]. Ocular metastases of breast cancer, although rare, can occur in both male and female patients, and in women breast is the most common site of origin of ocular metastatic tumors, since, in $49 \%$ of patients with ocular metastatic disease, the primary tumor origin was the breast [6].

Breast Cancer Ocular Metastatic Disease. Metastatic tumor accounts for the most common ocular malignancy [7]. The uveal tract is considered to be one of the most favored sites where breast cancer metastases develop [8]. The incidence of ocular breast cancer metastatic disease presents variations among different studies, with rates between 5 and $30 \%[9,10]$, attributed to the asymptomatic nature of ocular metastatic foci, in contrast to metastatic disease in other organs [11]. Metastatic foci of the disease in the lungs, central nervous system, or bones are usually detected prior to diagnosis of ocular metastases $[12,13]$. In fact, the only significant risk factor for the development of ocular malignant foci in patients with breast cancer is the dissemination of the disease in lungs and brain [11]. In rare cases ocular metastasis can represent the initial manifestation of an undiagnosed breast tumor [14]. The survival rate of patients with ophthalmic metastatic disease depends on the level of organ dysfunction caused by dissemination of the tumor [15].

\section{Clinical Signs and Manifestations}

Since the vast majority of ocular metastatic sites are created by hematogenous spread [16], the uveal tissue (iris, ciliary body, and choroid), especially the choroid, is the primary ocular site of breast cancer metastases. Involvement of the uvea occurs in up to $10 \%$ of cases with metastatic breast 


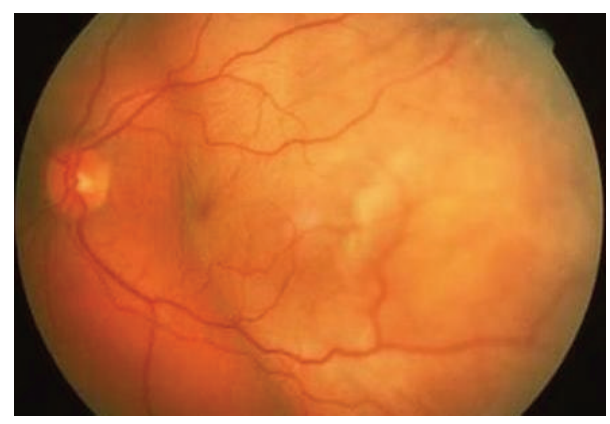

Figure 1: Fundus photo of the left eye of a patient demonstrating metastatic tumor involving the macula.

cancer: the highest metastatic efficiency index in comparison to all body tissues [17]. The vascularity of the choroid and microenvironmental factors have been proposed as possible explanations for increased metastatic dissemination in the choroid [18]. The choroid is predominantly affected with an incidence of $81 \%$ of ocular metastasis, followed by the iris with $9 \%$, optic disc with $5 \%$, and ciliary body with $2 \%$ [19]. The most common clinical complaint of patients with ocular metastatic disease is blurred vision, ocular pain, visual field defects, metamorphopsia, floaters, and photopsia [6]. In patients with diagnosed ocular metastatic disease, the risk of fellow eye involvement is estimated to be $5 \%$ in a period of ten months after diagnosis [20].

2.1. Symptoms and Signs of Metastatic Choroidal Disease. Most patients with metastatic choroidal disease $(81 \%$ of uveal metastases) are asymptomatic, and the most common symptom they develop is visual impairment with or without metamorphopsia [21]. The visual deterioration is caused by macular involvement of the malignant lesions (Figure 1), the presence of subretinal fluid in the fovea, or the induced hyperopia (Figure 1) [22]. Choroidal foci of metastases are typically located between the macula and the equator and are presented in fundoscopy as homogenous, creamy-yellow, plateau shaped lesions which spread laterally across the choroid [20]. In addition, they are usually complicated with serous foveal detachments and retinal pigment epithelium (RPE) alterations, appearing fundoscopically as deposits on the surface of the tumor with golden-brownish appearance (Figure 2) [23]. As in most metastatic diseases, tumors arising from breast cancer metastases are multifocal and are mainly located in the superotemporal quadrants of the fundus [22]. Choroidal metastases may also lead to an exudative retinal detachment, characterized by shifting patterns of subretinal fluid depending on the posturing of the patient (Figure 2) $[19,24]$.

2.2. Symptoms and Signs of Iris Metastasis. Clinical presentation of iris metastases (9\% of uveal metastases) includes ocular pain rather than visual impairment, if associated with secondary glaucoma or iridocyclitis [25]. The decreased visual acuity is secondary to anterior chamber seeding or to cataract formation. On slit lamp examination, they are identified as rapidly growing yellow to white solitary iris

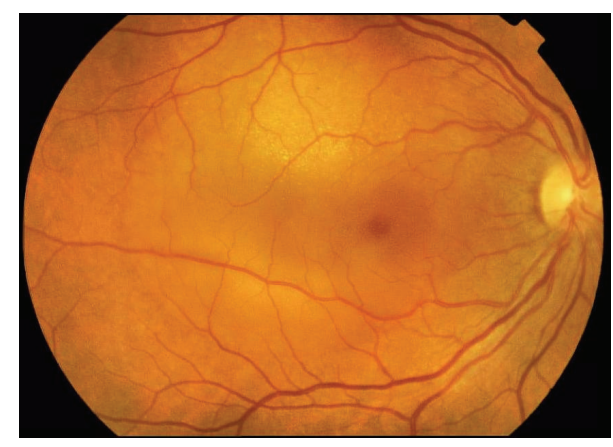

FIGURE 2: Fundus photo of the right eye of a patient with metastatic disease. Please note the tumor close to the macular area and the adjacent shallow retinal detachment involving the macula.

nodules with pupillary distortion, located in the iris (most commonly the midperiphery of the inferior quadrant) and in some cases infiltrating the trabecular meshwork $[26,27]$. More rarely they may be accompanied by pseudohypopyon [27].

2.3. Symptoms and Signs of Optic Disc Metastasis. Optic disc involvement is due either to direct extension of a choroidal tumor which is located close to the optic disc or to hematogenous spread of neoplastic cells to the circulation of the papilla [28]. Ophthalmoscopically, it appears as a diffuse yellow-white thickening of the optic nerve head in $84 \%$ of cases and as a distinct nodular lesion in $16 \%$, with minimal extension to the nerve fiber layer and secondary disc edema $[21,28]$.

\subsection{Symptoms and Signs of Metastatic Ciliary Body Disease.} Ciliary body metastases ( $2 \%$ of uveal metastases) are most commonly located in the inferior quadrant of the eye ( $25 \%$ of cases) and form cyst shaped or sessile masses, which are very difficult to be identified directly [29]. The ciliary body may be affected either directly or by an extension of a choroidal or iris tumor. Major complications of ciliary body masses include shallowing of the anterior chamber, cataract, and lens subluxation [24]. Common signs of ciliary body involvement are conjunctival and/or episcleral hyperemia, iridocyclitis, glaucoma, pseudohypopyon, and hyphaema [24].

\subsection{Extraocular Metastasis}

2.5.1. Orbital. The extraocular muscles represent the main site of breast cancer orbital metastasis, causing pain, proptosis, and diplopia. They are identified pathologically as solid deposits of the muscles [30]. Orbital metastasis may cause exophthalmos, from mass effect, or enophthalmos, when infiltrated muscles lead to posterior pulling of the eye $[31,32]$. Sporadic cases of breast cancer migration to the conjunctiva [30], the eyelids [33], and the cranial nerves [34] have been reported.

2.5.2. Cerebral. Brain is a common location for metastasis secondary to breast cancer, usually affecting the cortical 


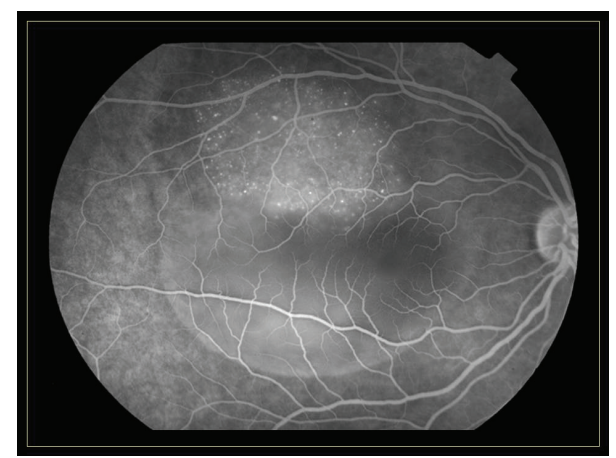

FIGURE 3: Fluorescein angiography of the same patient presented in Figure 2 .

and juxtacortical zones [35]. Clinical symptoms of brain insult include seizures, headache, and focal motor or mental syndromes [35]. As far as vision is concerned, patients may experience symptoms such as visual field defects like homonymous hemianopsia, and in certain cases dyschromatopsia $[36,37]$.

2.6. Paraneoplastic Manifestations. Paraneoplastic manifestations of breast cancer in the eye are not common. They are considered as immunological responses against the antigens of the tumor-expressed by normal cells-rather than metastatic processes [38]. They may cause diplopia, nystagmus, and loss of vision $[39,40]$.

\section{Diagnosis}

Diagnosis of ocular metastatic disease is mainly clinical. Slit lamp biomicroscopy is the hallmark for identifying metastatic sites, alongside history of breast cancer. Additional imaging studies are indicated in cases of doubt. More specifically, fluorescein angiography, fundus autofluorescence (FAF), and optical coherence tomography (OCT) are useful diagnostic tools for diagnosis of choroidal metastases (Figures 3-5) [41]. The fluorescein angiography reveals hyperfluorescence of the mass in the late venous phase [41]. Fundus autofluorescence provides tumor images as hyperfluorescent areas of focal pigmentation and subretinal fluid, with hypofluorescent margins [41]. Fourier-domain OCT demonstrates elevation of the retinal pigment epithelium (RPE) and retina, retinal thickening, and areas of retinal detachment, if present [41]. Additionally, B-scan ultrasonography demonstrates metastatic masses as areas with medium to high internal reflectivity [21]. The magnetic resonance imaging (MRI) contributes to differential diagnosis of breast cancer metastatic disease and choroidal melanoma, since choroidal melanoma demonstrates high signal intensity on T1-weighted images [42]. More recently, positron emission tomography-computed tomography scan (PET-CT scan) has been reported to contribute to identification of breast cancer choroidal metastatic disease [43].

Before deciding the exact treatment modalities that will be applied to each patient, it is essential to identify the primary source of the metastatic disease [44]. Although in most cases diagnosis of breast cancer is established at the time of ocular metastasis $[12,13]$, cases of ocular metastasis of unknown origin require histopathological confirmation with the performance of ocular tumor biopsy [45]. Moreover, intraocular biopsy may provide additional information about the nature of the tumor itself, contributing to the optimal therapeutic strategy. Specifically, intraocular biopsy may identify Her2/neu-positive patients that may benefit from adjuvant anti-Her2/neu therapy [46] and estrogen or progesterone receptor-positive tumor cells that would respond to endocrine therapy administration [47].

\section{Ocular Side Effects of Systemic Breast Cancer Treatment}

Therapeutic management of breast cancer involves systemic treatment [48] and/or local therapy [49]. Systemic therapy may lead to ocular tumor control as well, but in certain cases additional local treatment is required [48]. Systemic treatment includes hormone therapy, specifically selective estrogen receptor modulator (tamoxifen), aromatase inhibitors (anastrozole, letrozole, and exemestane), cytotoxic chemotherapy, or targeted therapy with monoclonal antibodies (trastuzumab) [50]. The aim of local treatment is to preserve patients' vision and improve their quality of life.

The treatment plan against ocular metastatic foci requires collaboration of ophthalmologists with oncologists and neuroradiologists, in order to define the optimal therapeutic approach for each patient, with the less possible side effects. Indications for ocular metastases treatment include visual deterioration due to metastatic tumors, location of metastases close to the macula or the optic nerve, enlargement of the neoplastic lesions despite systemic therapy, and lesions causing intolerant pain to patients [21].

4.1. Endocrine Treatment. The majority (60-70\%) of breast cancers in postmenopausal women express estrogen or progesterone receptors; thus they are susceptible to endocrine therapy [51]. The endocrine therapy consists of two regimens: selective estrogen receptor modulators (tamoxifen) and aromatase inhibitors.

4.1.1. Tamoxifen. Tamoxifen is a competitive antagonist of estrogen at its receptor site [52]; for premenopausal women with metastatic disease, tamoxifen is considered as the treatment of choice. Lower doses of tamoxifen $(20 \mathrm{mg} /$ day $)$ are currently used in order to avoid complications that have been reported in the past such as retinal toxicity (tamoxifen retinopathy: white, refractile deposits in nerve fiber and inner plexiform layers of the retina), optic neuritis, and corneal disease (tamoxifen keratopathy: "whorl-like" deposits on the cornea) and had led to discontinuation of the drug [52, 53].

4.1.2. Aromatase Inhibitors. In postmenopausal women, estrogens originate from adrenal androgen's peripheral conversion and aromatase inhibitors interfere in this path by preventing the conversion [52]. The third generation aromatase inhibitors (anastrozole, letrozole, and exemestane) 


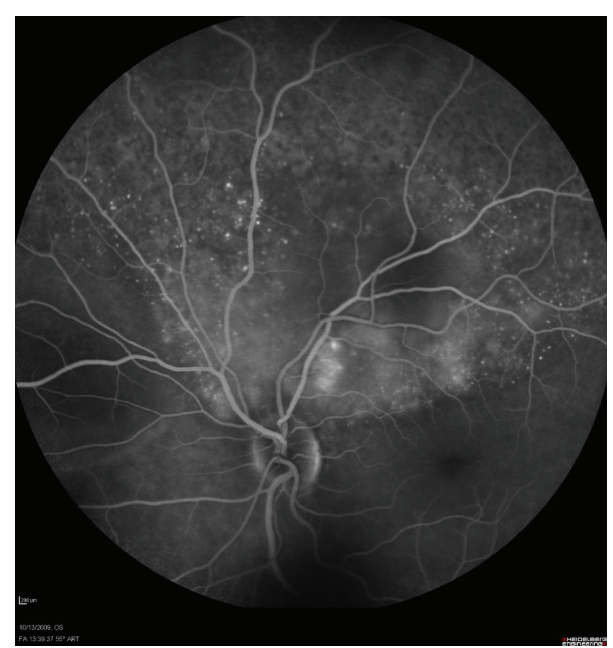

(a)

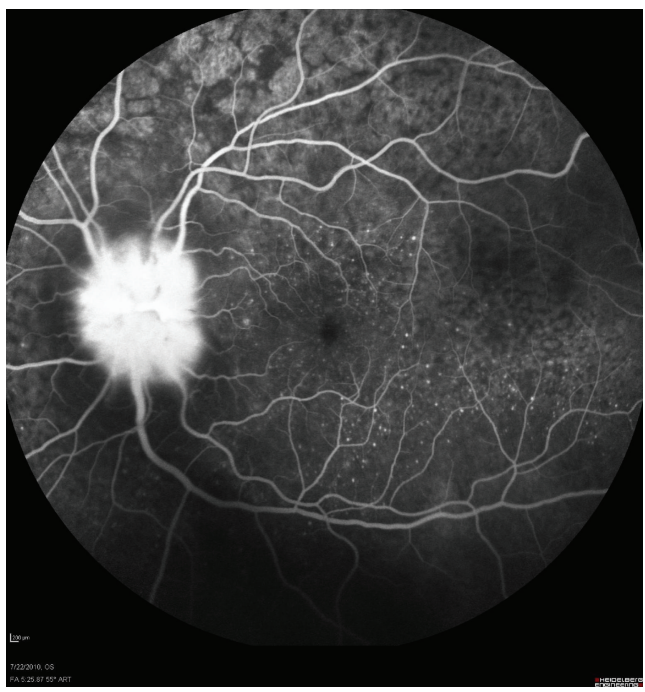

(c)

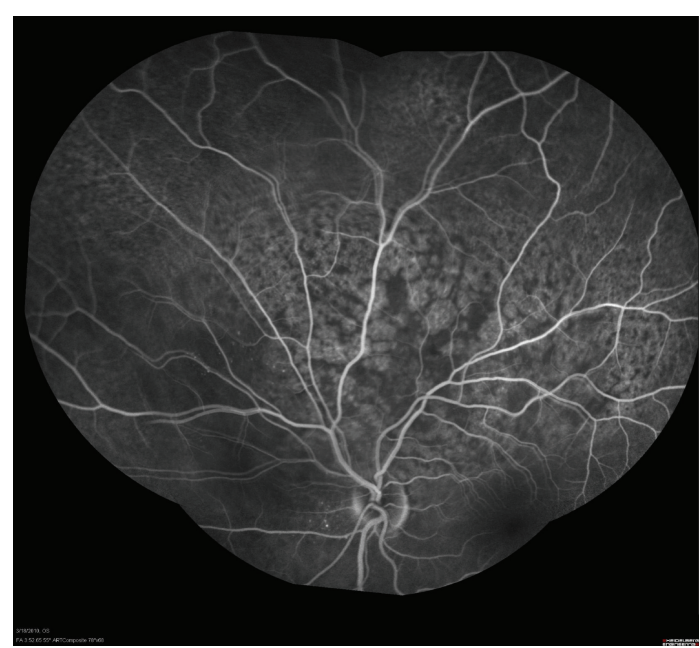

(b)

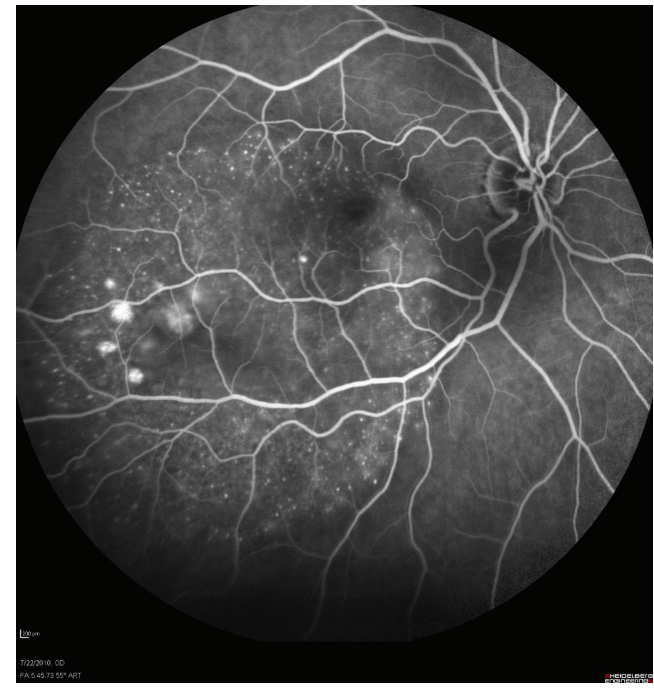

(d)

FIGURE 4: Angiographic imaging of a woman with metastatic breast cancer and choroidal disease. (a) Choroidal metastases presenting as pinpoint hyperfluorescence and dye pooling under the area of neurosensory detachment. (b) Resolution of angiographic signs after systemic chemotherapy. Retinal pigment epithelium alteration resembling "leopard skin" pattern. (c) Relapse of metastatic disease with infiltration of the optic disc. Diffuse late leakage in the peripapillary area. (d) Involvement of the fellow eye with small PEDs presenting pooling and numerous pinpoints.

have proven clinical efficacy in metastatic breast carcinoma [54]. Systemic side effects have been reported less commonly in comparison to tamoxifen and ocular toxicity has not been associated with the administration of these drugs [55].

4.2. Cytotoxic Treatment. The most commonly administered regimens are CMF (cyclophosphamide, methotrexate, and 5fluorouracil), FEC (5-fluorouracil, epirubicin, and cyclophosphamide), and AC (doxorubicin and cyclophosphamide) with or without the incorporation of taxanes such as docetaxel or paclitaxel [56]. Usual side effects of cytotoxic agents include blepharospasm [57], blurred vision [58], excessive lacrimation [59], and keratitis [60]. The symptoms usually appear up to 14 days after initiation of chemotherapy and do not last after the discontinuation of treatment [61].

4.3. Monoclonal Antibodies. Conventional chemotherapeutic modalities are targeted not only against neoplasmatic cells, but against normal cells as well, causing numerous side effects. This fact led to development of targeted therapies, which aim at molecular pathways involved in tumor cells proliferation [62]. The human epidermal growth factor receptor (HER2) is overexpressed in 25\% of breast cancer cases and is associated with more aggressive phenotype [62]. A humanized monoclonal antibody (trastuzumab), which binds to the extracellular juxtamembrane of this receptor, blocks the 


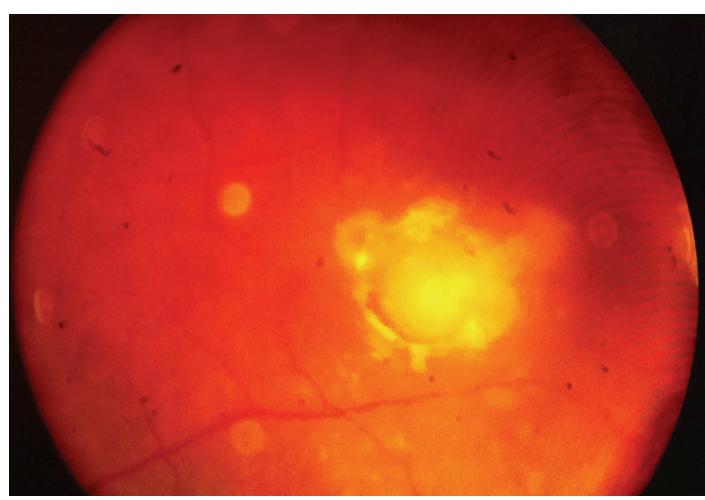

(a)

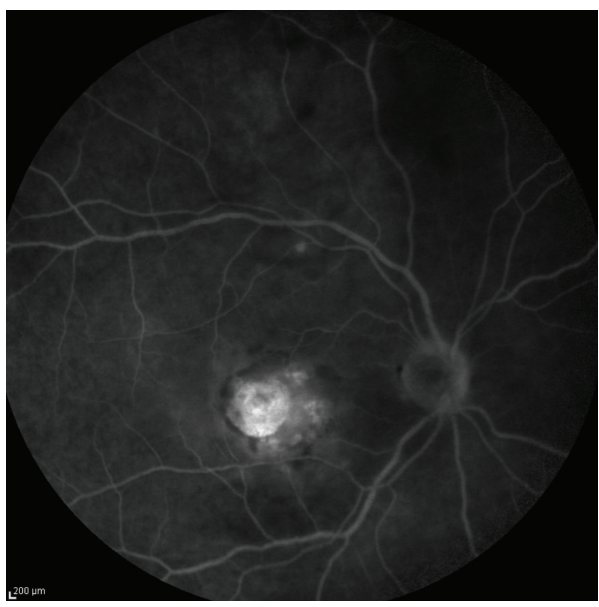

(c)

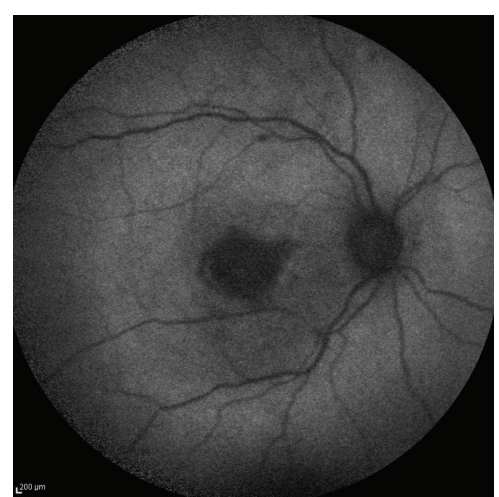

(b)

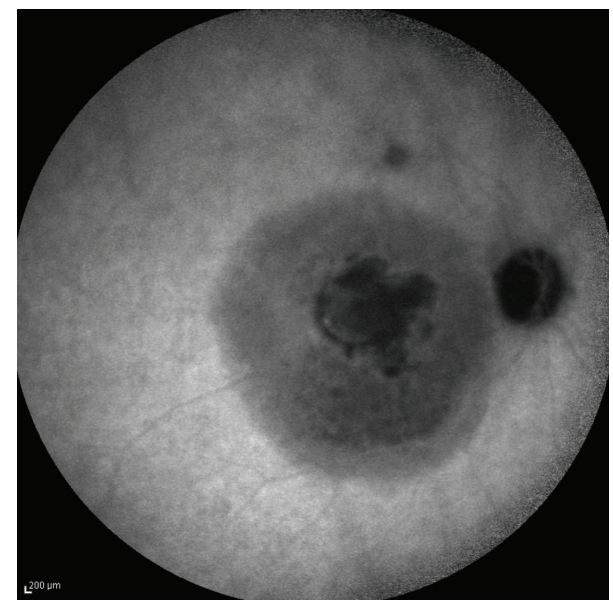

(d)

Figure 5: Infectious infiltration of the macula due to contamination with Staphylococcus epidermidis of venous fistula in a 45-year-old woman with breast cancer. (a) Creamy-white circular infiltration of the macula in a 45-year-old woman with breast cancer. (b) Fundus autofluorescence image with central defect. (c) Fluorescein angiography with staining of the central defect. (d) ICGA in late phase reveals a wider ring of retinal pigment epithelium involvement surrounding the central lesion.

activation of HER2. It has been reported that combined with other systemic therapies it has led to regression of ocular metastatic disease $[63,64]$. Ocular side effects of trastuzumab have not been reported.

\section{Local Management of Metastatic Eye Disease}

Local treatment's aim is to preserve patients' vision and improve their quality of life. It is administered in addition to systemic treatment regimens. Local treatment modalities include radiotherapy, laser application, intravitreal antivascular endothelial growth factors (anti-VEGF), photodynamic therapy, and enucleation of the eye.

5.1. Radiotherapy. External beam radiation therapy (EBRT) is the first and most widely applied local treatment modality against ocular metastatic disease [65]. It contributes to size regression of both choroidal and iris tumors, by damaging the DNA of the neoplasmatic cells [66]. Usual dosage applied varies between 20 and 50 grays. Regression of the metastatic tumor has been reported in $63-83 \%$ of cases [21]. EBRT is a treatment method which induces several ocular side effects. The most common ophthalmic complications are skin erythema, conjunctivitis, cataract formation, exposure keratopathy, iris neovascularization, and radiation retinopathy and papillopathy [67]. Patients' survival for over six months after EBRT application is the most important factor for developing side effects [68].

5.2. Alternative Radiation Treatment. Proton beam radiotherapy has been tested for localized intraocular irradiation [69]. Due to the nature of charged particles used, highly localized dose distribution is possible. Since protons deposit their energy at the end of their range, a lower number of radiation treatment fractions are needed, in comparison to EBRT. In addition, lower volume of irradiation is delivered to the surrounding tissues, resulting in less ocular toxicity than EBRT [70]. The results of the method were promising (tumor regression in $84 \%$ of cases) for improving patients' quality of life [69]. Side effects were reported in a lower rate than EBRT (29\%), most commonly cataract, keratitis, and radiation maculopathy/papillopathy [69]. 
5.3. Chemotherapy and Hormone Therapy. The application of systemic chemotherapy and hormonal therapy in patients with systematic, extraocular metastasis has demonstrated satisfactory results in managing metastatic eye disease, leading even to complete regression of choroidal metastasis [70]. Its efficacy, though, is only demonstrated in individual case reports and not through extensive trials.

5.4. Laser Treatment. Several types of lasers have been applied, with beneficial outcome against uveal metastatic disease [71]. Initially, transpupillary thermotherapy (TTT), in which a modified diode laser is used to deliver heat to choroid and RPE through the pupil, results in tumor necrosis [72]. In addition, laser photocoagulation with the use of argon or krypton causes occlusion of the vessels of the tumor which is followed by tumor necrosis [72]. It appears to be effective with no ocular complications, but it is applied in small sized metastatic foci [71].

5.5. Antivascular Endothelial Growth Factor-Targeted Treatment. Bevacizumab (Avastin) is a monoclonal antibody that blocks all VEGF-A isoforms. It was the first anti-VEGF therapy approved by the FDA for the treatment of colorectal, breast, and lung cancer [73]. Recent case series studies have shown promising results, since they demonstrated that bevacizumab is effective as a treatment option in patients with metastatic choroidal disease, unresponsive to systemic therapy. Its role is in preventing visual loss by achieving choroidal tumor regression [74]. Its efficacy is based on the antiangiogenic and antipermeability properties of bevacizumab [74]. More extensive trials are required for the establishment of the effectiveness and the determination of the exact treatment protocol.

5.6. Photodynamic Therapy. Photodynamic therapy (PDT) has lately been applied for the local regression of choroidal metastatic disease with promising outcomes, since its complete tumor control is reported in $78 \%$ of cases [75]. It achieves tumor destruction by producing highly reactive oxygen species and by activating immune response against tumor cells. In addition, the substance administered during PDT performance is verteporfin, which binds to vascular endothelial cells and leads to intraluminar thrombosis. The increased vascularity that characterizes the choroidal metastases explains the initial efficacy of the method [75].

5.7. Enucleation. Enucleation is only used as a treatment option for eyes with intolerable pain from secondary glaucoma development [23].

\section{Prognosis}

Despite recent advances in diagnosis and treatment modalities, the prognosis of breast cancer metastatic disease remains poor with estimated median life expectancy of 6 to 9 months [76].

\section{Conclusions}

Breast cancer is the most common malignancy in women. The incidence of breast cancer is increasing especially in developed world. Better screening, earlier detection, and better treatment modalities have positively altered the prognosis and survival time of patients suffering from breast cancer. This subsequently leads to an increased variety of ocular manifestations and problems associated with patients' vision that are likely to require ophthalmological consultation and management. Because early diagnosis and prompt management may positively alter the prognosis for these patients, ophthalmologists should be aware of this entity.

To date, the treatment of choice for ocular metastasis is radiotherapy. Chemotherapeutics also play an important role in the control of uveal metastasis. The use of anti-VEGF therapy is almost routine in the ophthalmology practice for many ocular diseases like age related macular degeneration, diabetic retinopathy. Its role in the treatment of uveal metastasis remains to be determined as more reports become available. However it may help avoid the need for other more damaging treatments.

\section{Disclaimer}

The authors alone are responsible for the content and writing of the paper.

\section{Conflict of Interests}

The authors report no conflict of interests.

\section{References}

[1] K. A. Ban and C. V. Godellas, "Epidemiology of breast cancer," Surgical Oncology Clinics of North America, vol. 23, no. 3, pp. 409-422, 2014.

[2] S. Dogan, F. Andre, and M. Arnedos, "Issues in clinical research for metastatic breast cancer," Current Opinion in Oncology, vol. 25, no. 6, pp. 625-629, 2013.

[3] S. H. Giordano, A. U. Buzdar, and G. N. Hortobagyi, "Breast cancer in men," Annals of Internal Medicine, vol. 137, no. 8, pp. 678-687, 2002.

[4] N. C. F. Hodgson, J. H. Button, D. Franceschi, F. L. Moffat, and A. S. Livingstone, "Male breast cancer: is the incidence increasing?" Annals of Surgical Oncology, vol. 11, no. 8, pp. 751755, 2004.

[5] K. Pant and U. Dutta, "Understanding and management of male breast cancer: a critical review," Medical Oncology, vol. 25, no. 3, pp. 294-298, 2008.

[6] M. I. Freedman and J. C. Folk, "Metastatic tumors to the eye and orbit. Patient survival and clinical characteristics," Archives of Ophthalmology, vol. 105, no. 9, pp. 1215-1219, 1987.

[7] A. P. Ferry and R. L. Font, "Carcinoma metastatic to the eye and orbit: I. A clinicopathologic study of 227 cases," Archives of Ophthalmology, vol. 92, no. 4, pp. 276-286, 1974.

[8] L. Weiss, "Analysis of the incidence of intraocular metastasis," British Journal of Ophthalmology, vol. 77, no. 3, pp. 149-151, 1993.

[9] C. C. Nelson, B. S. Hertzberg, and G. K. Klintworth, "A histopathologic study of 716 unselected eyes in patients with 
cancer at the time of death," American Journal of Ophthalmology, vol. 95, no. 6, pp. 788-793, 1983.

[10] K.-M. Kreusel, T. Wiegel, M. Stange, N. Bornfeld, and M. H. Foerster, "Intraocular metastasis in metastatic female breast cancer. Incidence, risk factors and therapy," Ophthalmologe, vol. 97, no. 5, pp. 342-346, 2000.

[11] T. Wiegel, K. M. Kreusel, N. Bornfeld et al., "Frequency of asymptomatic choroidal metastasis in patients with disseminated breast cancer: results of a prospective screening programme," British Journal of Ophthalmology, vol. 82, no. 10, pp. 1159-1161, 1998.

[12] R. Amer, J. Pe'er, I. Chowers, and I. Anteby, “Treatment options in the management of choroidal metastases," Ophthalmologica, vol. 218, no. 6, pp. 372-377, 2004.

[13] A. P. Ferry, "Metastatic carcinoma of the eye and ocular adnexa," International Ophthalmology Clinics, vol. 7, no. 3, pp. 615-658, 1967.

[14] G. Olfa, H. Riadh, T. Sameh, B. H. Fafani, and B. A. Slim, "Breast cancer discovered from choroidal metastasis: apropos of a case and review of the literature," Canadian Journal of Ophthalmology, vol. 44, no. 6, article e67, 2009.

[15] M. Amichetti, O. Caffo, E. Minatel et al., "Ocular metastases from breast carcinoma: a multicentric retrospective study," Oncology Reports, vol. 7, no. 4, pp. 761-765, 2000.

[16] A. P. Ferry and R. L. Font, "Carcinoma metastatic to the eye and orbit. I. A clinicopathologic study of 227 cases," Archives of Ophthalmology, vol. 92, no. 4, pp. 276-286, 1974.

[17] L. Weiss, "Comments on hematogenous metastatic patterns in humans as revealed by autopsy," Clinical \& Experimental Metastasis, vol. 10, no. 3, pp. 191-199, 1992.

[18] A. McCartney, "Intraocular metastasis," British Journal of Ophthalmology, vol. 77, no. 3, p. 133, 1993.

[19] J. A. Shields and C. L. Shields, "Metastatic tumors to the intraocular structures," in Intraocular Tumors, J. A. Shields and C. L. Shields, Eds., pp. 207-238, Saunders, Philadelphia, Pa, USA, 1992.

[20] H. Demirci, C. L. Shields, A.-N. Chao, and J. A. Shields, "Uveal metastasis from breast cancer in 264 patients," American Journal of Ophthalmology, vol. 136, no. 2, pp. 264-271, 2003.

[21] G. L. Kanthan, J. Jayamohan, D. Yip, and R. M. Conway, "Management of metastatic carcinoma of the uveal tract: an evidencebased analysis," Clinical and Experimental Ophthalmology, vol. 35, no. 6, pp. 553-565, 2007.

[22] L. Konstantinidis, I. Rospond-Kubiak, I. Zeolite et al., "Management of patients with uveal metastases at the Liverpool Ocular Oncology Centre," British Journal of Ophthalmology, vol. 98, no. 1, pp. 92-98, 2014.

[23] R. F. Stephens and J. A. Shields, "Diagnosis and management of cancer metastatic to the uvea: a study of 70 cases," Ophthalmology, vol. 86, no. 7, pp. 1336-1349, 1979.

[24] C. L. Shields, J. A. Shields, N. E. Gross, G. P. Schwartz, and S. E. Lally, "Survey of 520 eyes with uveal metastases," Ophthalmology, vol. 104, no. 8, pp. 1265-1276, 1997.

[25] J. J. Woog, J. Chess, D. M. Albert, D. K. Dueker, F. G. Berson, and J. Craft, "Metastatic carcinoma of the iris simulating iridocyclitis," British Journal of Ophthalmology, vol. 68, no. 3, pp. 167-173, 1984.

[26] Y. R. Barishak, E. Baruh, and M. Lazar, "Metastatic tumor of the iris: case report," Annals of Ophthalmology, vol. 10, no. 9, pp. 1191-1193, 1978.
[27] J. A. Shields, C. L. Shields, H. Kiratli, and P. de Potter, "Metastatic tumors to the iris in 40 patients," The American Journal of Ophthalmology, vol. 119, no. 4, pp. 422-430, 1995.

[28] J. A. Shields, C. L. Shields, and A. D. Singh, "Metastatic neoplasms in the optic disc: the 1999 Bjerrum lecture: Part 2," Archives of Ophthalmology, vol. 118, no. 2, pp. 217-224, 2000.

[29] W. E. Lieb, J. A. Shields, C. L. Shields, and G. L. Spaeth, "Mucinous adenocarcinoma metastatic to the iris, ciliary body, and choroid," British Journal of Ophthalmology, vol. 74, no. 6, pp. 373-376, 1990.

[30] R. Weiss, W. Grisold, K. Jellinger, J. Mühlbauer, W. Scheiner, and M. Vesely, "Metastasis of solid tumors in extraocular muscles," Acta Neuropathologica, vol. 65, no. 2, pp. 168-171, 1984.

[31] A. van der Heijden, A. Twijnstra, W. P. M. A. Lamers, P. S. G. J. Hupperets, and G. Freling, "An unusual cause of diplopia in a cancer patient," European Journal of Cancer, vol. 27, no. 10, pp. 1315-1316, 1991.

[32] J. W. Henderson, Orbital Tumors, WB Saunders, Philadelphia, $\mathrm{Pa}, \mathrm{USA}, 1973$.

[33] G. J. Zhang, I. Adachi, D. F. Yin et al., "Eyelid metastasis from breast cancer showing marked response to chemotherapy," Japanese Journal of Clinical Oncology, vol. 25, no. 1, pp. 10-15, 1995.

[34] J. D. Bullock and B. Yanes, "Ophthalmic manifestations of metastatic breast cancer," Ophthalmology, vol. 87, no. 10, pp. 961973, 1980.

[35] R. Massot-Punyet, J. Almajano, and J. M. Camacho, "Cerebral metastases," Revista de Neurologia, vol. 31, no. 12, pp. 1242-1247, 2000.

[36] J. Aharon-Peretz and M. Feinsod, "Visual hemineglect as presenting symptom of right parietal tumors," Harefuah, vol. 126, no. 1, pp. 5-56, 1994.

[37] G. J. Green and S. Lessell, "Acquired cerebral dyschromatopsia," Archives of Ophthalmology, vol. 95, no. 1, pp. 121-128, 1977.

[38] A. S. Polans, D. Witkowska, T. L. Haley, D. Amundson, L. Baizer, and G. Adamus, "Recoverin, a photoreceptor-specific calcium-binding protein, is expressed by the tumor of a patient with cancer-associated retinopathy," Proceedings of the National Academy of Sciences of the United States of America, vol. 92, no. 20, pp. 9176-9180, 1995.

[39] C. Budde-Steffen, N. E. Anderson, M. K. Rosenblum et al., "An antineuronal autoantibody in paraneoplastic opsoclonus," Annals of Neurology, vol. 23, no. 5, pp. 528-531, 1988.

[40] P. W. Wirtz, P. A. E. S. Smitt, J. I. Hoff et al., "Anti-Ri antibody positive opsoclonusmyoclonus in a male patient with breast carcinoma," Journal of Neurology, vol. 249, no. 12, pp. 1710-1712, 2002.

[41] S. Natesh, K. J. Chin, and P. T. Finger, "Choroidal metastases fundus autofluorescence imaging: correlation to clinical, OCT, and fluorescein angiographic findings," Ophthalmic Surgery Lasers and Imaging, vol. 41, no. 4, pp. 406-412, 2010.

[42] J. A. Smith, E. S. Gragoudas, and E. B. Dreyer, "Uveal metastases," International Ophthalmology Clinics, vol. 37, no. 4, pp. 183-199, 1997.

[43] S. Solav, R. Bhandari, A. Sowani, and S. Saxena, "Choroidal metastasis from carcinoma of breast detected on F18-FDG PET CT scan: a case report and review of literature," Indian Journal of Nuclear Medicine, vol. 25, no. 4, pp. 160-163, 2010.

[44] K. Hemminki, M. Bevier, J. Sundquist, and A. Hemminki, "Sitespecific cancer deaths in cancer of unknown primary diagnosed with lymph node metastasis may reveal hidden primaries," 
International Journal of Cancer, vol. 132, no. 4, pp. 944-950, 2012.

[45] N. Eide and L. Walaas, "Fine-needle aspiration biopsy and other biopsies in suspected intraocular malignant disease: a review," Acta Ophthalmologica, vol. 87, no. 6, pp. 588-601, 2009.

[46] A. C. Wolff, M. E. H. Hammond, J. N. Schwartz et al., "American Society of Clinical Oncology/College of American Pathologists guideline recommendations for human epidermal growth factor receptor 2 testing in breast cancer," Archives of Pathology and Laboratory Medicine, vol. 131, no. 1, pp. 18-43, 2007.

[47] M. E. H. Hammond, D. F. Hayes, M. Dowsett et al., "American society of clinical oncology/college of american pathologists guideline recommendations for immunohistochemical testing of estrogen and progesterone receptors in breast cancer," Journal of Clinical Oncology, vol. 28, no. 16, pp. 2784-2795, 2010.

[48] G. N. Hortobagyi, "Treatment of breast cancer," The New England Journal of Medicine, vol. 339, no. 14, pp. 974-984, 1998.

[49] C. L. Shields, J. A. Shields, P. De Potter et al., "Plaque radiotherapy for the management of uveal metastasis," Archives of Ophthalmology, vol. 115, no. 2, pp. 203-209, 1997.

[50] C. J. Chen, A. N. McCoy, J. Brahmer, and J. T. Handa, "Emerging treatments for choroidal metastases," Survey of Ophthalmology, vol. 56, no. 6, pp. 511-521, 2011.

[51] C. K. Osborne, "Tamoxifen in the treatment of breast cancer," New England Journal of Medicine, vol. 339, no. 22, pp. 1609-1618, 1998.

[52] M. E. Manquez, M. M. Brown, C. L. Shields, and J. A. Shields, "Management of choroidal metastases from breast carcinomas using aromatase inhibitors," Current Opinion in Ophthalmology, vol. 17, no. 3, pp. 251-256, 2006.

[53] S. G. Nayfield and M. B. Gorin, "Tamoxifen-associated eye disease: a review," Journal of Clinical Oncology, vol. 14, no. 3, pp. 1018-1026, 1996.

[54] J. Berry, "Are all aromatase inhibitors the same? A review of controlled clinical trials in breast cancer," Clinical Therapeutics, vol. 27, no. 11, pp. 1671-1684, 2005.

[55] M. Baum, A. U. Buzdar, J. Cuzick et al., "Anastrozole alone or in combination with tamoxifen versus tamoxifen alone for adjuvant treatment of postmenopausal women with early breast cancer: first results of the ATAC randomised trial," The Lancet, vol. 359, no. 9324, pp. 2131-2139, 2002.

[56] J. Bergh, P.-E. Jönsson, B. Glimelius, P. Nygren, and SBU-group. Swedish Council of Technology Assessment in Health Care, "A systematic overview of chemotherapy effects in breast cancer," Acta Oncologica, vol. 40, no. 2-3, pp. 253-281, 2001.

[57] H. D. Weiss, M. D. Walker, and P. H. Wiernik, "Neurotoxicity of commonly used antineoplastic agents: (first of two parts)," The New England Journal of Medicine, vol. 291, no. 2, pp. 75-81, 1974.

[58] G. Kende, S. R. Sirkin, P. R. M. Thomas, and A. I. Freeman, "Blurring of vision. A previously undescribed complication of cyclophosphamide therapy," Cancer, vol. 44, no. 1, pp. 69-71, 1979.

[59] P. S. Imperia, H. M. Lazarus, and J. H. Lass, "Ocular complications of systemic cancer chemotherapy," Survey of Ophthalmology, vol. 34, no. 3, pp. 209-230, 1989.

[60] G. Bonadonna, E. Brusamolino, P. Valagussa et al., "Combination chemotherapy as an adjuvant treatment in operable breast cancer," The New England Journal of Medicine, vol. 294, no. 8, pp. 405-410, 1976.
[61] A. D. Letson, F. H. Davidorf, and R. A. Bruce Jr., "Chemotherapy for treatment of choroidal metastases from breast carcinoma," American Journal of Ophthalmology, vol. 93, no. 1, pp. 102-106, 1982.

[62] C. A. Hudis, "Trastuzumab-mechanism of action and use in clinical practice," The New England Journal of Medicine, vol. 357, no. 1, pp. 39-51, 2007.

[63] E. Munzone, F. Nolè, G. Sanna, and A. Goldhirsch, "Response of bilateral choroidal metastases of breast cancer to therapy with trastuzumab," Breast, vol. 14, no. 5, pp. 380-383, 2005.

[64] Z. W. Wong, S. J. Phillips, and M. J. Ellis, "Dramatic Response of Choroidal Metastases from Breast Cancer to a Combination of Trastuzumab and Vinorelbine," Breast Journal, vol. 10, no. 1, pp. 54-56, 2004.

[65] J. I. Lim and Z. Petrovich, "Radioactive plaque therapy for metastatic choroidal carcinoma," Ophthalmology, vol. 107, no. 10, pp. 1927-1931, 2000.

[66] R. V. Paul Chan and L. H. Young, "Treatment options for metastatic tumors to the choroid," Seminars in Ophthalmology, vol. 20, no. 4, pp. 207-216, 2005.

[67] S. B. Rudoler, B. W. Corn, C. L. Shields et al., "External beam irradiation for choroid metastases: identification of factors predisposing to long-term sequelae," International Journal of Radiation Oncology Biology Physics, vol. 38, no. 2, pp. 251-256, 1997.

[68] T. Wiegel, D. Bottke, K.-M. Kreusel et al., "External beam radiotherapy of choroidal metastases-final results of a prospective study of the German Cancer Society (ARO 95-08)," Radiotherapy and Oncology, vol. 64, no. 1, pp. 13-18, 2002.

[69] E. K. Tsina, A. M. Lane, D. N. Zacks, J. E. Munzenrider, J. M. Collier, and E. S. Gragoudas, "Treatment of metastatic tumors of the choroid with proton beam irradiation," Ophthalmology, vol. 112, no. 2, pp. 337-343, 2005.

[70] M. E. Manquez, C. L. Shields, E. C. Karatza, and J. A. Shields, "Regression of choroidal metastases from breast carcinoma using aromatase inhibitors," British Journal of Ophthalmology, vol. 89, no. 6, pp. 776-777, 2005.

[71] S. Levinger, S. Merin, R. Seigal, and J. Peer, "Laser therapy in the management of choroidal breast tumor metastases," Ophthalmic Surgery and Lasers, vol. 32, no. 4, pp. 294-299, 2001.

[72] H. Kiratli and S. Bilgiç, "Transpupillary thermotherapy in the management of choroidal metastases," European Journal of Ophthalmology, vol. 14, no. 5, pp. 423-429, 2004.

[73] J. C. Yang, L. Haworth, R. M. Sherry et al., "A randomized trial of bevacizumab, an anti-vascular endothelial growth factor antibody, for metastatic renal cancer," The New England Journal of Medicine, vol. 349, no. 5, pp. 427-434, 2003.

[74] V. Fenicia, S. Abdolrahimzadeh, G. Mannino, S. Verrilli, M. Balestrieri, and S. M. Recupero, "Intravitreal bevacizumab in the successful management of choroidal metastases secondary to lung and breast cancer unresponsive to systemic therapy: a case series," Eye, vol. 28, no. 7, pp. 888-891, 2014.

[75] S. Kaliki, C. L. Shields, S. A. Al-Dahmash, A. Mashayekhi, and J. A. Shields, "Photodynamic therapy for choroidal metastasis in 8 cases," Ophthalmology, vol. 119, no. 6, pp. 1218-1222, 2012.

[76] V. Ratanatharathorn, W. E. Powers, J. Grimm et al., "Eye metastasis from carcinoma of the breast: diagnosis, radiation treatment and results," Cancer Treatment Reviews, vol. 18, no. 4, pp. 261-276, 1991. 


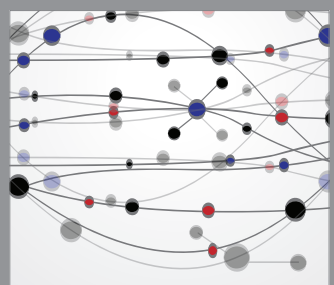

The Scientific World Journal
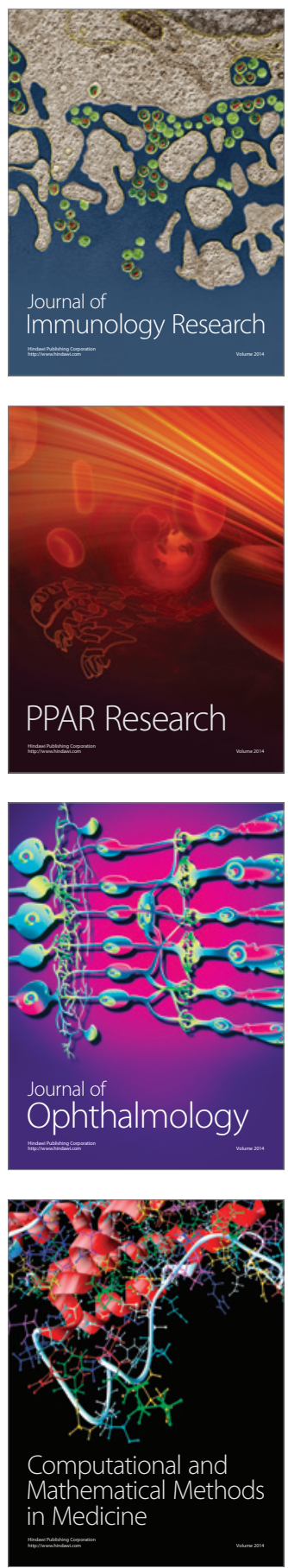

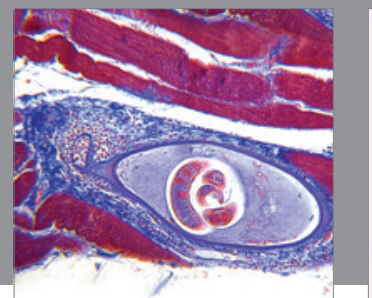

Gastroenterology

Research and Practice
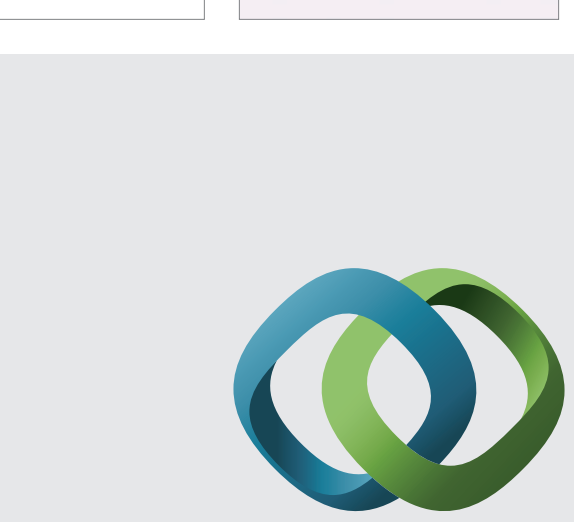

\section{Hindawi}

Submit your manuscripts at

http://www.hindawi.com
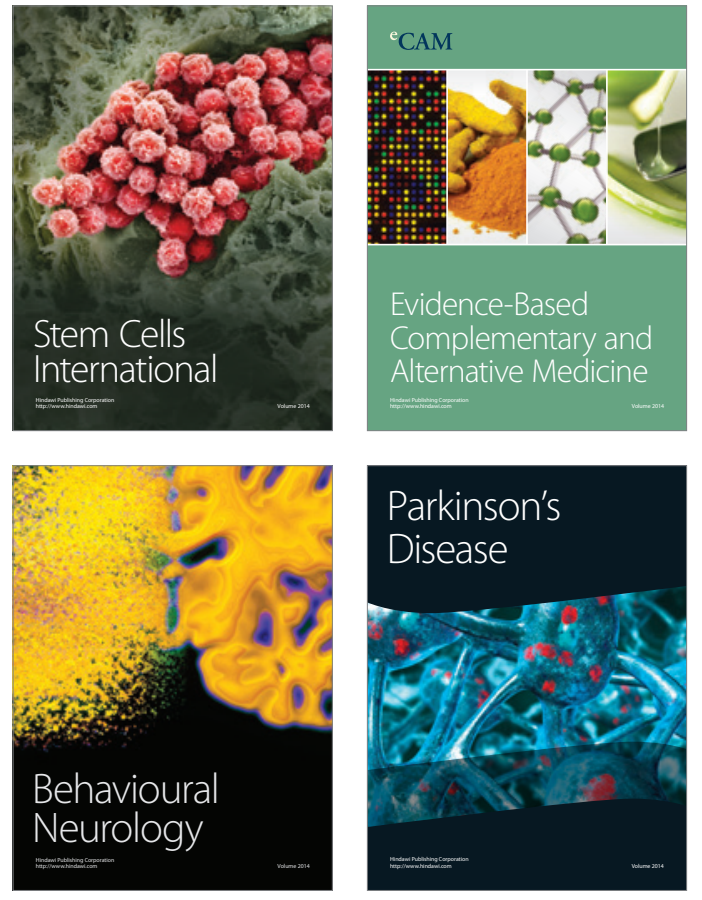
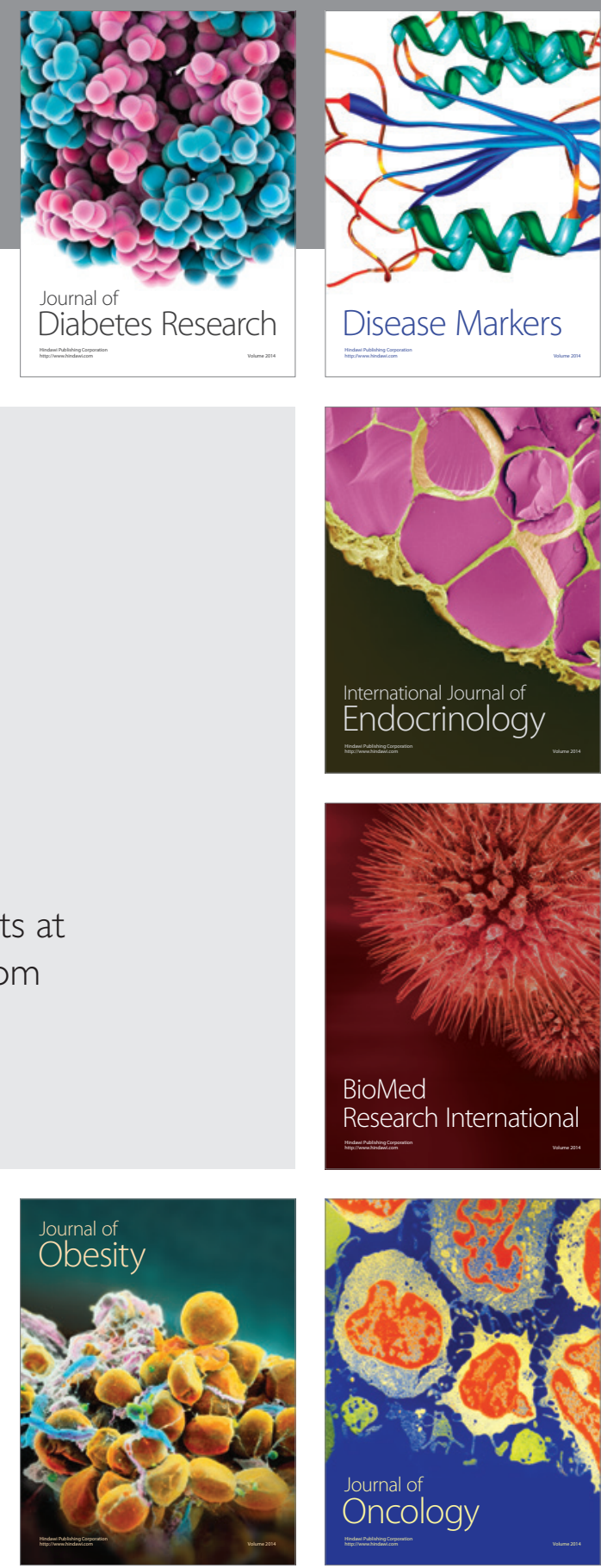

Disease Markers
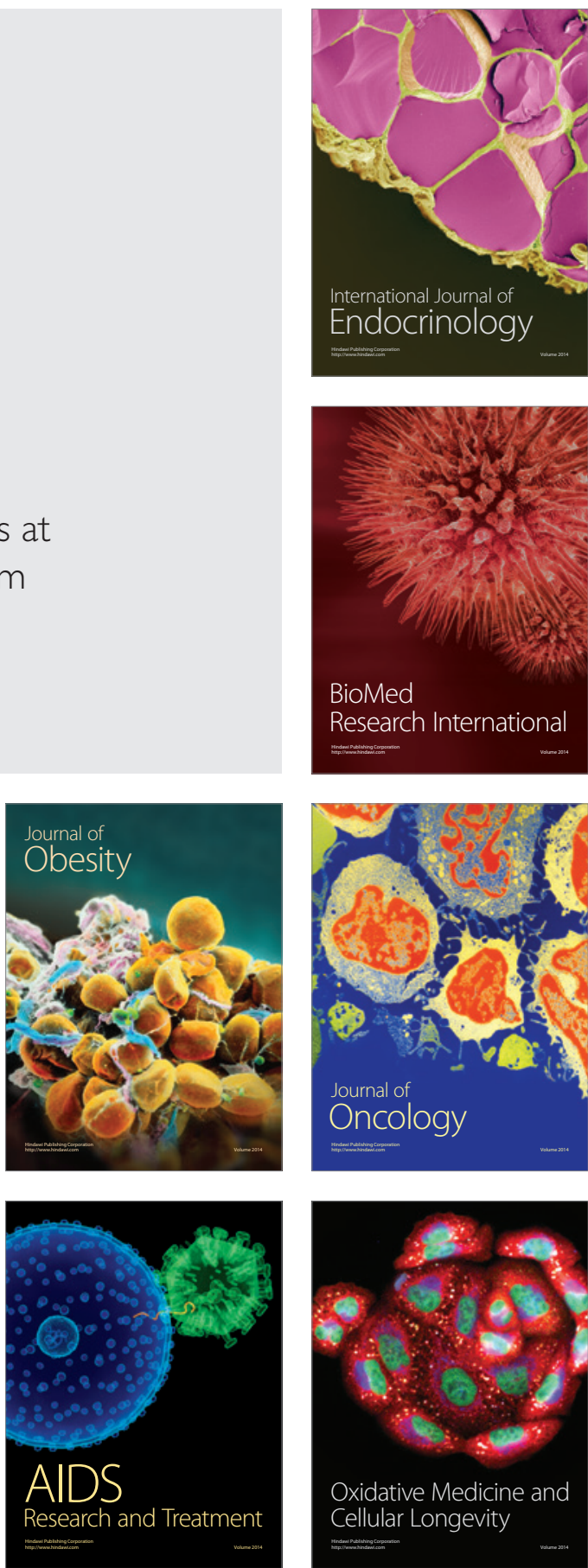